\title{
Influence of time dependent flows on the threshold of the kinematic dynamo action
}

\author{
A. de la Torre ${ }^{\mathrm{a}}$, J. Burguete ${ }^{\mathrm{b}}$, and C. Pérez-García (†) \\ Departamento de Física y Matemática Aplicada, Universidad de Navarra, Irunlarrea s/n, \\ 31008 Pamplona
}

\begin{abstract}
A numerical study of the influence of slowly evolving velocity fields in the threshold of the dynamo action is presented. Using experimental time averaged velocity fields, harmonic variations are introduced in a kinematic code in order to characterize the response of the magnetic field to a broad range of frequencies. A critical frequency is found around $\omega_{c}=200$ where a transition is obtained. For large values of the frequency (i.e. smaller periods) the magnetic field can not see the velocity fluctuations and the response of the system corresponds to that of the mean flow. For smaller frequencies, the magnetic field sees the slow evolution of the velocity field, and reduces significatively its growth rates when compared to the mean value. This loss of efficiency is due to the dissipation that appears during the transition between the magnetic eigenvectors corresponding to each one of the velocity fields.
\end{abstract}

\section{Introduction}

The dynamo action in a conducting fluid is the generating mechanism of magnetic fields in astrophysical bodies [1]. Although the theoretical framework can be stablished very easily from Maxwell equations $[2,3]$ the properties of real materials and their flows make the analysis of these equations very difficult. In the magnetohydrodynamic (MHD) approximation (i.e. flow velocities much smaller than the velocity of light) and for an incompressible and neutral conducting fluid, the evolution equations can be simplified, and only two are needed, the induction and Navier-Stokes equations:

$$
\begin{gathered}
\frac{\partial \mathbf{B}}{\partial t}=\nabla \times(\boldsymbol{u} \times \mathbf{B})+\eta \nabla^{2} \mathbf{B} \\
\frac{\partial \mathbf{u}}{\partial t}+(\mathbf{u} \cdot \nabla) \mathbf{u}=-\frac{1}{\rho} \nabla p+\nu \nabla^{2} \mathbf{u}+\frac{1}{\rho \mu_{0}}(\nabla \times \mathbf{B}) \times \mathbf{B}+\frac{1}{\rho} \mathbf{F}_{e x t}
\end{gathered}
$$

where $\mathbf{u}$ and $\mathbf{B}$ are the velocity and magnetic fields, and $\nu$ and $\eta$, properties of the considered material (respectively, the kinematic viscosity and the magnetic diffusivity $\eta=\left(\mu_{0} \sigma_{c}\right)^{-1}$, $\sigma_{c}$ electrical conductivity of the fluid). The continuity equation $(\nabla \cdot \mathbf{u}=0)$ and Gauss law $(\nabla \cdot \mathbf{B}=0)$ close the equation system.

The boundary conditions are crutial in MHD problems. For example, they affect the way the magnetic energy is diffused: for perfectly conducting walls the magnetic field is confined inside the cavity, while for isolant walls the magnetic field can diffuse outside. As the dynamo action is a transfer from kinetic to magnetic energy, different boundary conditions lead to very different thresholds and even anihilation of the dynamo process.

\footnotetext{
a e-mail: admonguio@alumni.unav.es

b e-mail: javier@fisica.unav.es
} 
In the induction equation the first term transforms kinetic into magnetic energy, while the last term is responsible for the diffusion (Ohm's law). It is interesting to note that this equation is linear in $\mathbf{B}$, as the term $\boldsymbol{\nabla} \times(\mathbf{u} \times \mathbf{B})$ can be rewritten as $(\mathbf{B} \cdot \boldsymbol{\nabla}) \mathbf{v}-(\mathbf{v} \cdot \boldsymbol{\nabla}) \mathbf{B}$. The retroaction of the magnetic field on the velocity field is recovered through the Lorentz force (in equation (2) the electric current $\mathbf{J}$ has been replaced using the relationship $\mathbf{J}=\boldsymbol{\nabla} \times \mathbf{B}$ ). Finally, in any dynamo experiment or natural system, an external force $\mathbf{F}_{\text {ext }}$ has to be applied to sustain the system out of thermodynamic equilibrium: these forces can be convective, Coriolis forces, or, in the case of experiments, produced by propellers. Depending on the time scales involved in the dynamo action and in the advection, a distinction is done in the dynamo mechanisms: fast (dynamo time scale and advective $L / U$ times are similar) and slow (advective time is much smaller than the dynamo time scale) $[4,5]$.

The equations can be adimensionalized in such a way that only two parameters govern the evolution: the hydrodynamic Reynolds number, $R e=U L / \nu$ and the magnetic Reynolds number $R m=U L / \eta$ being $U$ and $L$ characteristic velocity and lengths.

For the known liquid metals, the magnetic Prandtl number $P m=R m / R e$ is around $10^{-5}$. The minimum requirement to achieve dynamo action is that shear and dissipation terms in equation (1) are of the same order of magnitude, i.e. $R m \simeq 1$. Then, even for a very simple MHD system, the hydrodynamics is always turbulent $\left(R e \gtrsim 10^{5}\right)$. That makes the analytical and numerical tools useless, and the experiments become decisive. In the last years there has been a growing interest in this field, and different experiments around the world have been carried out. Two of them were succesful in 2000 [6-9], but in very confined geometries that restrict the retroaction of the magnetic field over the velocity field. Some years ago a new experiment was proposed in a von Kármán flow configuration, with very promising results $[10,11]$. Very recently $[12,13]$ a new setup of this configuration has demonstrated the feasibility of the dynamo action in homogeneous flows.

All these experiments require a previous analysis of the attainable flows that can be tested. Because of the high risks involved in sodium experiments, the classical approach is to measure a velocity field in a harmless material, like water, with hydrodynamical properties similar to molten sodium. This velocity field is introduced as a parameter in the induction equation and the threshold of this flow is obtained performing numerical simulations [14,15]. This analysis is called the kinematic dynamo approach.

Most of the times the flow is simplified, in order to deal with a more affordable problem. Some of the simplifications relay on the symmetries, both spatial and temporal, of the velocity field: usually, the flow is supposed to be stationary, and sometimes even axisymmetric. Using this approach, the dynamo threshold can be obtained with a very accurate precission in most of the situations [6-9].

Nevertheless, it has been shown that these assumptions in some cases are no longer valid [16] The averaged flow can break different symmetries of the problem, becoming no longer stationary, but time-dependent. The effect of time dependent flows has been studied deeply along the last decades. In 1992 Galloway and Proctor proposed a time evolving flow with chaotic paths that was able to produce a fast dynamo $[17,18]$. Other flows where the different scales interact have been proposed, making diffuse the frontier between fast and slow dynamos [19]. Recently, other time dependent flows have been introduced to model the turbulence of real flows [20], the effect of a modulated Ponomarenko flow [21]-[23] or simple model flows of large eddies evolution [24]-[26]. Other works have analyzed the effect of periodic time-dependent flows in a sphere [27]. Concerning experiments, to our knowledge only one experimental work carried out in the Karlsruhe facility has presented results on modulated flows [28].

The purpose of this paper is to present recent results about the influence of time-dependent flows on the dynamo threshold in a von Kármán swirling flow. The spatial symmetries of the problem will be preserved, although it is known that they are broken [16,29]. These flows have been studied analytically $[30,31]$, numerically $[32,33]$ and experimentally $[14,15,34]$. In the next section we present the experimental setup and the velocity fields that have been obtained. The third section includes a brief description of the numerical scheme and the numerical results. The results will be discussed in the fourth section. 
(a)

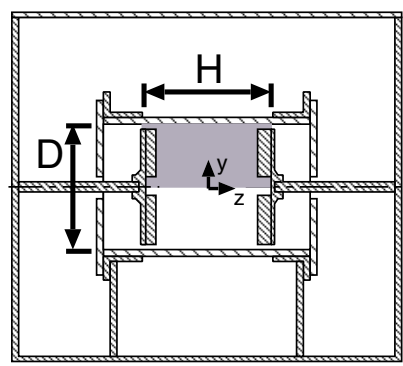

(c)

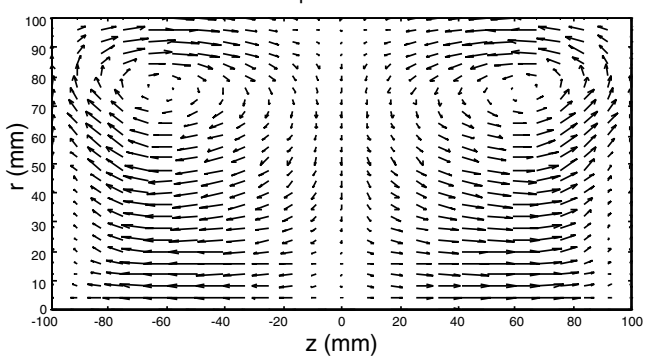

(b)

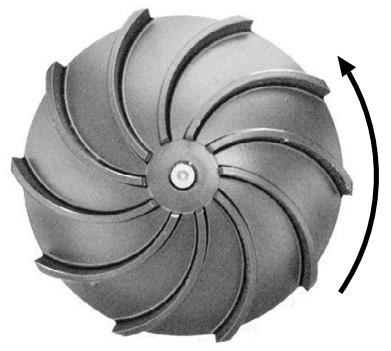

(d) $\quad v_{\text {tor }}\left(v_{\theta}\right)$

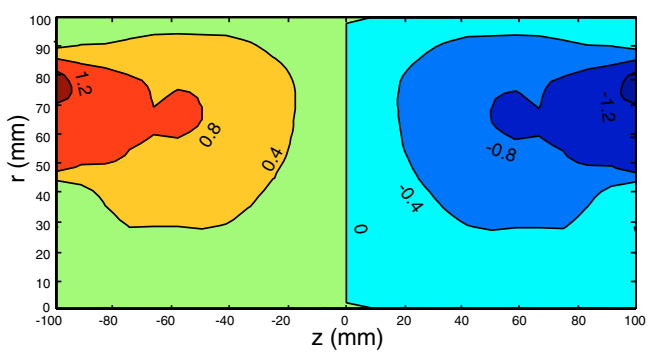

Fig. 1. Experimental setup. (a) Horizontal cylinder with the propellers, inside the tank. The north propeller is at $z=H / 2$ and the south is at $z=-H / 2$. (b) Photograph of the propeller used in the flow presented in figures $(\mathrm{c}, \mathrm{d})$. The rotation sense with the convex side sets the azimuthal velocity as positive in the south propeller. These blades have a radius of $5 \mathrm{~cm}$ and are $2 \mathrm{~cm}$ high. Symmetrized velocity field $\mathbf{V}_{\mathbf{B}}$ (see explanation in the text): (c) stream-vectors $V_{r}, V_{z}$ in the plane $\theta=\pi / 2$ and (d) contourplot of $V_{\theta}$. Figures (c) and (d) where obtained in the grey region in figure (a).

\section{Experimental von Kármán flow}

We generate a von Kármán flow by stirring the fluid inside a cylindrical vessel (figure 1(a)). Two propellers are placed at both ends of the cylinder. The separation between the propellers determines the heigth of the experimental volume. The parameter $\Gamma$, defined as the ratio between the height $H$ (which can be modified) and the diameter $D$ of the cylinder (fixed, $D=20 \mathrm{~cm}$ ), can be modified continuously in the range $[0,2.5]$. The data presented in this work have been obtained for $\Gamma=1$ (that is, fixing $H$ to $20 \mathrm{~cm}$ ). The cylinder is placed inside a tank of $150 \mathrm{l}$ of volume in order to avoid optical problems with the different interfaces and to assure the temperature stability. The fluid used is water at $21^{\circ} \mathrm{C}$.

Different propellers have been used: disks with blades or flat disks. All of them have a radius of $R_{\text {prop }}=8.75 \mathrm{~cm}$. The blades are used to increase the transmission of energy to the fluid (figure 1(b)). Both propellers are rotated counterclockwise by two motors of $1.5 \mathrm{~kW}$ power, which allows a rotation regime in the range $f=0-20 \mathrm{~Hz}$. Although each motor is independent, in this experiment the frequencies of both propellers are the same, in what is called the exact counter-rotation regime.

The Reynolds number $R e=R V_{\text {prop }} / \nu$ is defined using as typical lenght scale the radius of the cylinder and as typical velocity scale the propeller's rim velocity, $V_{\text {prop }}=2 \pi f R_{\text {prop }}$. This number can be varied continuously in the range $R e \sim 10^{3}-10^{6}$. At these high $R e$ the flow is in a fully developed turbulence regime.

The measurement of the velocity field is performed with two complementary techniques: A LDV system (with a chosen spatial resolution of $1 \mathrm{~cm}$ and temporal resolution up to $100 \mathrm{kHz}$ ) and a PIV system (spatial resolution of $1 \mathrm{~mm}$ and temporal resolution of $15 \mathrm{~Hz}$ ). The LDV system allows the measurement of two components of the instantaneous velocity field $\mathbf{v}=\left(v_{r}, v_{\theta}, v_{z}\right)$. Using the standard cylindrical coordinate system of figure 1(a), the components measured are $v_{\theta}$ and $v_{z}$. The mean flow $\mathbf{V}=\langle\mathbf{v}\rangle$ is obtained by averaging the measurements for more than 300 times the period of the propeller and deducing the radial component $V_{r}$ by mass 
conservation. The PIV measurements gives the instantaneous field $v_{r}, v_{z}$ in the plane $\theta=\pi / 2$, and no traces of the mean flow are observed. This is due to the high turbulence rate ( $r m s$ value over the mean value) which vary between $60-150 \%$, depending on the spatial position and the velocity component measured. Averaging the velocity field for long times, this mean velocity field is consistent with the LDV mean velocity field.

A typical mean flow obtained in this experiment $\left(R e=3 \times 10^{5}\right)$ is in figure $1(\mathrm{c})-(\mathrm{d})$. The propellers drag the flow, breaking it into two cells with opposite azimuthal velocity. In each cell the flow is absorbed through the axis, and expelled radially at the propellers. The circulation is closed returning to the shear layer by the walls, where the fluid is dragged to the axis of the cylinder.

As the averaging time is much larger than any characteristic time of the dynamics of the flow [16], the mean flow obtained in this way is axisymmetric $\left(R_{\theta}\right.$ symmetry, i.e. $\left.\mathbf{V} \neq \mathbf{V}(\theta)\right)$. Another imposed symmetry is the $R_{\pi}$ symmetry, in which the mean flow is invariant under a $\pi$ rotation around any diameter in the $z=0$ plane. These assumptions are known not to hold in some configurations $[16,32]$. The $R_{\theta}$ symmetry is broken when coherent structures (vortices) or modulation in the shear layer are found, while the $R_{\pi}$ symmetry fails when the shear layer is displaced from the equatorial plane.

Nevertheless, in this paper we will focus only on the time dependent character of the velocity field, so both symmetries will be imposed. First assuming the independence of $\mathbf{V}$ on $\theta$, and second splitting the field into a symmetric and an antisymmetric part, neglecting this last element. The influence of this spatial symmetry breaking will be presented elsewhere.

\section{Numerical simulations}

The numerical simulations are done using a kinematic dynamo scheme, i.e, the velocity field has been measured in a water experiment and introduced as a parameter in the induction equation (1). The numerical code is pseudospectral, with Fourier modes in both the azimuthal and axial directions and finite differences in the radial component. Thus, the considered volume is an infinite cylinder, with insulating boundary conditions in the radial direction. The temporal scheme is a single-step semi-implicit mixed Adams-Bashforth/Adams-Moulton of second order. A detailed explanation of the numerical scheme and the boundary conditions can be found in references $[14,35]$.

The procedure to evaluate the influence of time dependent flows is as follows: A harmonic evolution is inserted in the code between two static mean velocity fields, $\mathbf{V}_{\mathbf{A}}$ and $\mathbf{V}_{\mathbf{B}}$, obtained using different propellers (the velocity field presented in figure 1(c)-(d) corresponds to $\mathbf{V}_{\mathbf{B}}$ ). Although the topology (i.e. spatial positions of the maximum velocity in the toroidal component and of the stagnation point in the poloidal component) are very similar and both produce dynamo action, their effectiveness is very different, as the respective thresholds are $R m_{A} \sim 85$ and $R m_{B} \sim 140$. This very different behaviour of comparable experimental velocity fields is very well known[14,15].

The harmonic variation of the velocity field is defined as:

$$
\mathbf{V}_{\boldsymbol{\omega}}(t)=\mathbf{V}_{\mathbf{S}}+\mathcal{A}_{\text {mod }} \mathbf{V}_{\mathbf{D}} \cos (\omega t)=\frac{\mathbf{V}_{\mathbf{A}}+\mathbf{V}_{\mathbf{B}}}{2}+\mathcal{A}_{\text {mod }} \frac{\mathbf{V}_{\mathbf{A}}-\mathbf{V}_{\mathbf{B}}}{2} \cos (\omega t)
$$

where $\omega$ is varied in the range $\left[10^{-1}, 10^{3}\right]$. This is an artificial condition, as each one of these fields is stationary because of the measurement procedure. Nevertheless, this approach allows us to check the response of the magnetic field to different forcing frequencies $\omega$, using velocity fields whose effect can be easily evaluated.

The adimensionalizations used for the characteristic length and time are the container radius $R$ and the magnetic diffusion time $\tau_{B}=R^{2} / \eta$ respectively. In an hipothetical experiment with the setup used in this work $(R=10 \mathrm{~cm})$ but using sodium the characteristic time will be $\tau_{B}=10^{-3} \mathrm{~s}$. Thus, an adimensonal frequency $\omega=100$ will correspond to a real value of $f_{\text {exp }} \sim 16 \mathrm{KHz}$. 

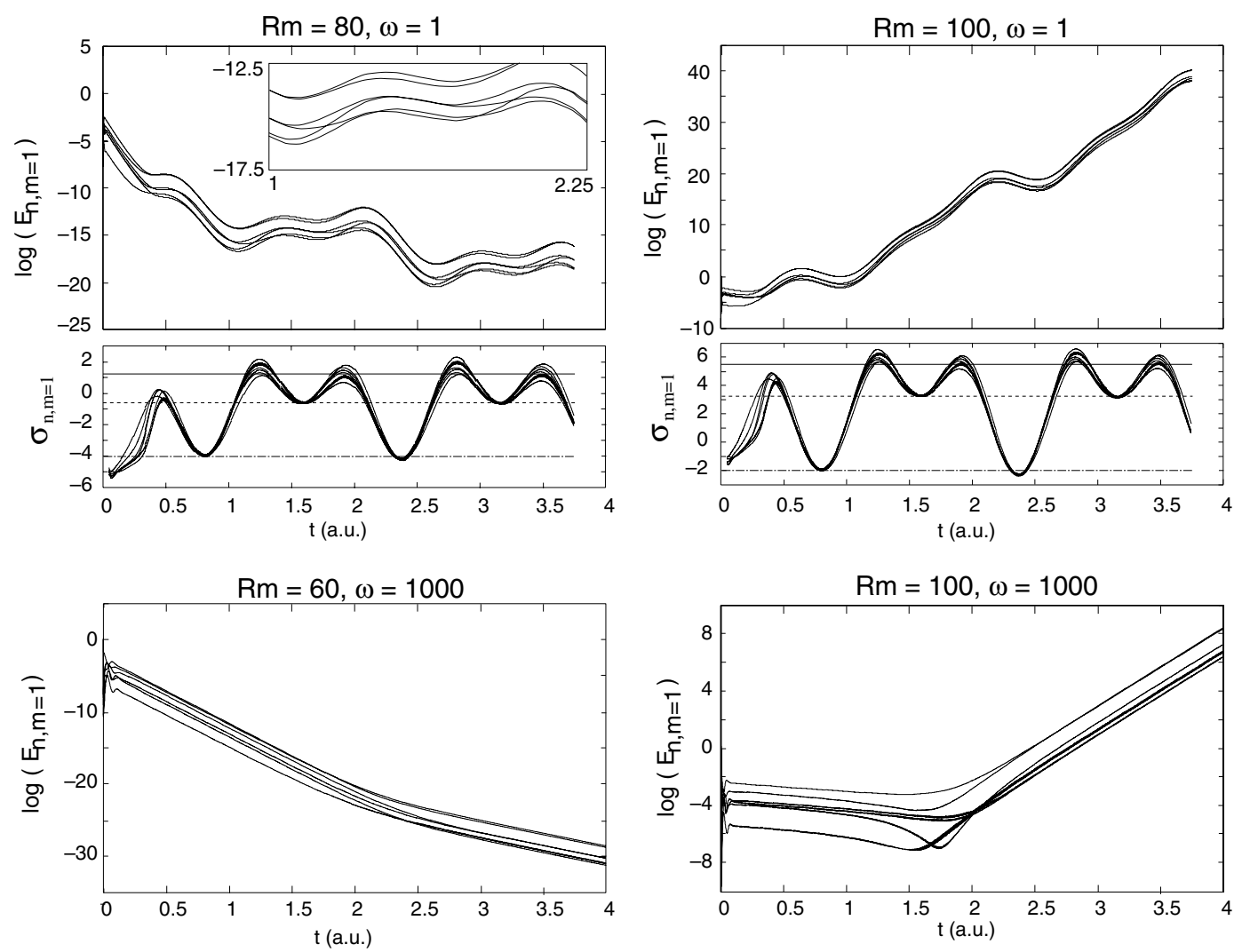

Fig. 2. Top row: Energy evolution (up) and instanteneous growth rate $\sigma_{n, m}$ (bottom) for frequencies $\omega=1$ and $R m=80$ (below threshold, left) and $R m=100$ (threshold reached, right). Bottom row: Energy evolution for frequency $\omega=1000$ and $R m=60$ (left) and $R m=100$ (right). The evolution for $t \lesssim 1.5$ corresponds to the transient period.

Both velocity fields $\mathbf{V}_{\mathbf{A}, \mathbf{B}}$ are normalized to the same maximum value (once adimensionalized, $\left.\left|\mathbf{V}_{\mathbf{A}, \mathbf{B}}\right| \leq R m\right)$. The parameter $\mathcal{A}_{\text {mod }}$ allows to increase the modulation for a given set of velocity fields $\mathbf{V}_{\mathbf{A}, \mathbf{B}}$ and can be modified at will. In this work we will present results concerning the case $\mathcal{A}_{\text {mod }}=1$. Further work varying this parameter is under run. The intensity of the modulation can be defined as $\mathcal{I}_{\text {mod }}=\mathcal{A}_{\text {mod }}\left[\max \left\{\mathbf{V}_{\mathbf{D}}\right\} / \max \left\{\mathbf{V}_{\mathbf{S}}\right\}\right]$. The value of this parameter for the velocity fields presented here is $\mathcal{I}_{\text {mod }}=0.66$.

Due to the temporal evolution defined in equation (3), we cannot use the classical definition of $R m$, as it becomes time-dependent. We will use a $R m$ definition based on the behaviour of the velocity filed in a period $T=2 \pi / \omega$

$$
R m=\max _{0 \leq t<T}\left\{V_{\omega}(t)\right\} R / \eta
$$

As the objective is the study of the dynamo action, in the numerical runs we look at the evolution of the magnetic energy of the different modes:

$$
\begin{gathered}
\mathbf{B}(\mathbf{r}, t)=\sum_{n, m} \mathbf{b}_{n, m}(r) \exp [i(m \theta+n 2 \pi z / H)] \\
E=\frac{1}{\mu_{0}} \int_{V}|\mathbf{B}|^{2} P \mathrm{~d} v=\frac{1}{\mu_{0}} \int_{V} \sum_{n, m}\left|\mathbf{b}_{n, m}(r)\right|^{2} \mathrm{~d} v=\sum_{n, m} E_{n, m} \\
E_{m, n}=\frac{1}{\mu_{0}} \int_{V}\left|\mathbf{b}_{m, n}(r)\right|^{2} \mathrm{~d} v \sim e^{\sigma_{n, m} t}
\end{gathered}
$$




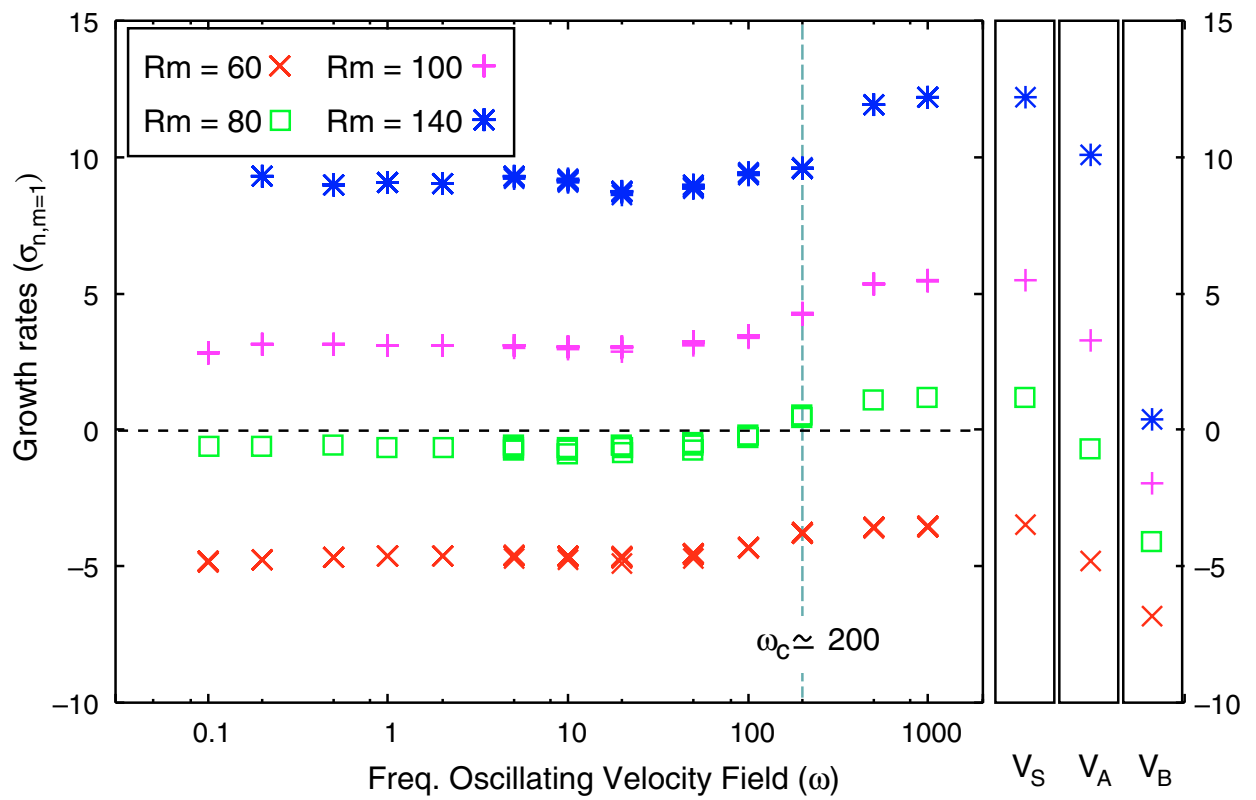

Fig. 3. Growth rates $\left\langle\sigma_{n, m=1}\right\rangle v s$. frequency for different $R m$. The critical frequency $\omega_{c} \simeq 200$ that divides the frequency range into two plateaus with different growth rates is plotted as a vertical dashed line. The corresponding growth rates for the time-averaged velocity fields $\mathbf{V}_{\mathbf{A}}, \mathbf{V}_{\mathbf{B}}$ and $\mathbf{V}_{\mathbf{S}}$ are provided on the right for comparison purposes.

For stationary velocity fields, the dynamo action is reached when one of the growth rates $\sigma_{n, m}$ becomes positive. But in these numerical simulations, because of the time dependence of the velocity field, this criterium can not be used. For example, in figure 2, top right, the energy for $R m=100$ and $\omega=1$ is clearly growing, but the instantaneous growth rate is negative for some time intervals. Then, our criterium is that the dynamo action is reached when:

$$
\left\langle\sigma_{n, m}\right\rangle=\frac{1}{T} \int_{T=\frac{2 \pi}{\omega}} \sigma_{n, m}(t) \mathrm{d} t>0
$$

for some $n, m$.

\section{Results and discussion}

Different runs have been done varying the magnetic Reynolds number (60 to 140) and the frequency $\left(10^{-1}\right.$ to $\left.10^{3}\right)$. The results are summarized in figures 2 and 3 .

The averaged growth rate increases with $R m$ in all the cases: the injection term is enlarged compared to the diffusion term. The critical $R m$ where the dynamo action is reached depends on the frequency of the velocity field (see figure 3): for $\omega>200, R m_{c}$ is around 75 , but for $\omega<200, R m_{c}$ is around 85. Then, the dynamo threshold increases when the frequency is diminished. This can be seen in a different way: for a constant value of $R m$ (i.e, $R m=80$, figure 3 ) the growth rate changes from positive (dynamo action for $\omega>200$ ) to negative (no dynamo for $\omega<200$ ).

In the frequency domain two different regimes are found. For large $\omega$ (figure 2 bottom row) the growth rate is nearly constant and an exponential evolution of the energy is obtained once the transient time is over. For small frequencies (figure 2 top) the growth rate is time dependent with a quasi-periodic behaviour, oscillating between the growth rates $\sigma_{\mathbf{A}}, \sigma_{\mathbf{B}}$ and $\sigma_{\mathbf{S}}$ that correspond to the dominant velocity field $\mathbf{V}_{\mathbf{A}}, \mathbf{V}_{\mathbf{B}}$ (horizontal dashed lines in figure 2, top row) or $\mathbf{V}_{\mathbf{S}}$ (continuous horizontal line). As the topology of these fields are slightly different, 
the topology of the magnetic field evolves accordingly (see relative amplitudes of the different modes $n$ in the inset of figure 2, top row, left).

Using the definition of $\left\langle\sigma_{n, m}\right\rangle$ given in equation (8), the growth rates can be compared for different frequencies and $R m$ (figure 3). Again, two different regimes with a borderline in $\omega_{c}=200$ can be distinguished. For large frequencies $(L F)$ the magnetic field has a growth rate $\sigma_{L F}$ similar to that of $\mathbf{V}_{\mathbf{S}}$, while below that border the growth rates are always smaller $\sigma_{S F}<\sigma_{L F}$.

This behaviour can be understood by the modification of the topology of $\mathbf{B}$. The velocity fields $\mathbf{V}_{\mathbf{A}}$ and $\mathbf{V}_{\mathbf{B}}$ are very similar, but some differences remain: the spatial position of maximum azimuthal velocity and of the stagnation point in the $\left(v_{r}, v_{z}\right)$ plane differ in a $10 \%$ of the radius. When the forcing time scale $T=2 \pi / \omega$ is very large, the magnetic field is slaved to the velocity field $\mathbf{V}_{\omega}(t)$. Then, the magnetic field $\mathbf{B}$ will oscillate between the instantanous solutions of the induction equation, so the resulting averaged growth rate $\left\langle\sigma_{n, m}\right\rangle$ is smaller than that of the averaged velocity field $\mathbf{V}_{\mathbf{S}}$. This linkage is preserved until $T$ is smaller than $(0.10 R)^{2} / \eta$, the time that $\mathbf{B}$ needs to evolve between the corresponding eigenvectors. That means that above a frequency of around $\omega_{c}=2 \pi\left(\eta /(0.10 R)^{2}\right) \simeq 100$ (adimensionalized) the magnetic field will only perceive the mean velocity field $\mathbf{V}_{\mathbf{S}}$ but not the fast evolving part $\mathbf{V}_{\mathbf{D}} \cos (\omega t)$. This $\omega_{c}$ is expected to be field dependent, and numerical simulations are under work to test this assertion.

Nevertheless, the growth rate for small frequencies $\sigma_{S F}$ should always be different from the average velocity field growth rate $\sigma_{\mathbf{S}}$ that will be reached for large frequencies $\sigma_{L F}=\sigma_{\mathbf{S}}$. One question that arise naturally is whether this effect will be observed in a real experiment. According to the choosen adimensionalization, the small frequency regime $\left(\omega \leq \omega_{c}=200\right)$ corresponds to $f_{\text {exp }} \leq 32 \mathrm{kHz}$ for $R=0.1 \mathrm{~m}$ cylindrical vessels or $f_{\text {exp }} \leq 8 \mathrm{kHz}$ for $R=0.2 \mathrm{~m}$. As the experimental flows may present slowly evolving coherent structures [16], an increase of the experimental dynamo action threshold should be expected compared to kinematic dynamo simulations based on averaged stationary flows.

Finally, the difference $\Delta \sigma=\sigma_{L F}-\sigma_{S F}$ depends on the value of $\mathcal{I}_{\text {mod }}$. Obviously, it should vanish when $\mathcal{I}_{\text {mod }} \rightarrow 0$, but for large values it is not clear what the relationship between $\Delta \sigma$ and $\mathcal{I}_{\text {mod }}$ will be.

\section{Conclusion}

In this paper we have presented the effect of time-dependent flows on the threshold of the dynamo action. Two different regimes have been obtained, depending on the frequency of the velocity field applied. These two regions are connected through a region where the averaged growth rate changes smoothly from $\sigma_{S F}$ to $\sigma_{L F}$ centered around $\omega_{c} \simeq 200$.

For large frequencies the magnetic field can not follow the fast fluctuations and it behaves as if only the $\mathbf{V}_{\mathbf{S}}$ were applied. An exponential growth of the energy is obtained. For small frequencies (i.e. large period of the harmonic oscillation of the velocity field) the magnetic field is slaved to the flow. The magnetic energy growth rate evolves in time, oscillating between the growth rates of each velocity field $\mathbf{V}_{\mathbf{A}}$ and $\mathbf{V}_{\mathbf{B}}$. In this region the averaged growth rate is smaller than that obtained for large frequencies, due to diffusion processes.

In a real MHD experiment the velocity field has different time scales (i.e. a real turbulent flow, where coherent structures as vortices can appear). In such a flow the slow scales can not be neglected and the dynamo action threshold can be increased significantly. Although in this paper we have used flows as real as posible, the modulation with a sigle frequency is an artifact that can not be achieved in any real experiment. Actually, all the time scales appear simultaneously in real flows.

Finally, some questions remain unanswered. One is the combined effect of both timeevolution and spatial symmetry breaking. It has been shown [16] that in this configuration a complex dynamics between two states (asymmetric flows) is possible. A similar behaviour could be obtained in a hypotethical MHD experiment and magnetic field reversals could be observed due to the transtition between these mirrored hydrodynamic states. Other questions are related to the response of the system to arbitrary velocity field evolution, instead of harmonic. 
We are grateful to Jacques Léorat, who provided the original code and kindly answered to all our requests about MHD numerical simulations.

This work has been done with finantial support from the Spanish government (research projects FIS2004-06596-C02-01 and UNAV05-33-001) and University of Navarra (PIUNA program). One of us, AdlT, thanks the "Asociación de Amigos" for a post-graduate grant.

We would like to dedicate this work to Carlos Perez-Garcia that deceased on July 31st, 2005, just when this project was starting. This was the last project in which he was involved and he could not see the final results. We are in debt with him, because he always enjoyed revealing to us and to other people the details of complex systems.

\section{References}

1. J. Larmor, Rep. Brit. Assoc. Adv. Sci., 159 (1919)

2. H.K. Moffatt, Magnetic Field Generation in Electrically Conducting Fluids (Cambridge University Press, Cambridge, 1978)

3. R. Moreau, Magnetohydrodynamics (Kluwer Academic Publishers, Dortrecht, 1990)

4. S.I. Vainshtein, Ya.B. Zeldovich, Sov. Phys. Usp. 75, 159 (1972)

5. H.K. Moffatt, Nature 341, 285 (1989)

6. A. Gailitis, O. Lielausis, S. Dement'ev, E. Placatis, A. Cifersons, G. Gerbeth, T. Gundrum, F. Stefani, M. Christen, H. Hänel, G. Will, Phys. Rev. Lett. 84, 4365 (2000)

7. A. Gailitis, O. Lielausis, S. Dement'ev, E. Placatis, A. Cifersons, G. Gerbeth, T. Gundrum, F. Stefani, M. Christen, H. Hänel, G. Will, Phys. Rev. Lett. 86, 3024 (2001)

8. R. Stieglitz, U. Müller, Naturwissenschaften 87, 381 (2000)

9. R. Stieglitz, U. Müller Phys. Fluids 13, 561 (2001)

10. M. Bourgoin, L. Marié, F. Pétrélis, J. Burguete, A. Chiffaudel, F. Daviaud, S. Fauve, P. Odier, J.-F. Pinton, Phys. Fluids 14, 3046 (2002)

11. F. Pétrélis, M. Bourgoin, L. Marié, J. Burguete, A. Chiffaudel, F. Daviaud, S. Fauve, P. Odier, J.-F. Pinton, Phys. Rev. Lett. 90, 174501 (2003)

12. R. Monchaux et al., Phys. Rev. Lett. 98, 044502 (2007)

13. M. Berhanu et al., Europhys Lett. (2007) (submitted)

14. L. Marie, J. Burguete, F. Daviaud, J. Leorat, Eur. Phys. J B 33, 469 (2003)

15. F. Ravelet, A. Chiffaudel, F. Daviaud, J. Leorat, Phys. Fluids 17, 117104 (2005)

16. A. de la Torre, J. Burguete, Phys. Rev. Lett. [arXiv:physics/0702151] (submitted)

17. D.J. Galloway, M.R.E. Proctor, Nature 356, 691 (1992)

18. R. Hollerbach, D.J. Galloway, M.R.E. Proctor 74, 3145 (1995)

19. F. Cattaneo, S.M. Tobias, Phys. Fluids 17, 127105 (2005)

20. J. Lorat, Magnetohydrodynamics 31, 367 (1995)

21. Yu. B. Ponomarenko, J. Appl. Mech. Tech. Phys. 14, 755 (1972)

22. C. Normand, Phys. Fluids 15, 1606 (2003)

23. M. Peyrot, F. Plunian, C. Normand, Phys. Fluids (2007) (submitted)

24. F. Pétrélis, S. Fauve, Europhys. Lett. 76, 602 (2006)

25. S. Fauve, F. Pétrélis, Peyresq Lectures on Nonlienar Phenomena, edited by J. Sepulchre (World Scientific, Singapore, 2003), pp. 1-64

26. J.P. Laval, P. Blaineau, N. Leprovost, B. Dubrulle, F. Daviaud, Phys. Rev. Lett. 96, 204503 (2006)

27. A.P. Willis, D. Gubbins, Geophys. Astrophys. Fluid Dynam. 98, 537 (2004)

28. U. Müller, R. Stieglitz and S. Horanyi, F. Busse, XXI ICTAM (CDROM Proceedings) ISBN 8389687-01-1 (IPPT-PAN, Warsaw, 2004)

29. F. Ravelet, L. Marie, A. Chiffaudel, F. Daviaud, Phys. Rev. Lett. 93, 164501 (2004)

30. T. von Kármán, Z. Angew. Math. Mech. 1, 233 (1921)

31. P. Zandbergen, D. Dijkstra, Ann. Rev. Fluid Mech. 19, 465 (1987)

32. C. Nore, L.S. Tuckerman, O. Daube, S. Xin, J. Fluid Mech. 477, 51 (2003)

33. C. Nore, L.M. Witkowski, E. Foucault, J. Pecheux, O. Daube, P. Le Quere, Phys. Fluids 18, 054102 (2006)

34. C. Nore, F. Moisy, L. Quartier, Phys. Fluids 17, 064103 (2005)

35. J. Lorat, Prog. Ser. Am. Inst. Astronaut. Aeronaut. 162, 282 (1994) 